\title{
Commentaire
}

\section{Le rôle des médecins dans la prévention des féminicides au Canada}

\author{
Nori L. Bradley MD MSc Kin MSc QSP, Nada Gawad MD MÉd, Jaclyn McNamara BScA JD, Najma Ahmed MD PhD
}

Citation : CMAJ 2021 December 6;193:E1844-5. doi : 10.1503/cmaj.211324-f

Voir la version anglaise de l'article ici : www.cmaj.ca/lookup/doi/10.1503/cmaj.211324

A u Canada, une femme est assassinée tous les 2,5 jours; de 144-178 féminicides ont été commis chaque année entre 2015 et $2019^{1,2}$, et en 2021, la tendance s'est accentuée ${ }^{3}$. Depuis 2015, dans $90 \%$ des cas, une personne a été inculpée et $90 \%$ des accusés étaient des hommes. Parmi les femmes assassinées, 50\% l'ont été par leur conjoint et $26 \%$ par un membre de leur famille ${ }^{1,4}$. La fin d'une relation n'annule pas le risque de décès auquel une femme est exposée : 20\%-22\% des féminicides ont été perpétrés par d'exconjoints au cours des 18 mois suivant la séparation ${ }^{5,6}$.

Les femmes comptent pour $80 \%$ des cas déclarés de violence conjugale et le phénomène touche tous les groupes d'âge, toutes les races ou ethnicités et toutes les classes socio-économiques ${ }^{7}$. Les femmes les plus à risque sont jeunes (15-24 ans), immigrantes, réfugiées, autochtones ou vivant avec un handicap. En outre, les données sur les féminicides au Canada montrent une tendance alarmante chez les femmes autochtones vivant en zone non urbaine ${ }^{4}$. De 2016 à 2019, les femmes vivant en zone non urbaine ont représenté $42 \%$ des féminicides au Canada, alors que seulement $16 \%$ de la population canadienne vit à l'extérieur des villes, et le quart de toutes les femmes assassinées au Canada sont autochtones ${ }^{2,5}$. En 2020, la proportion des féminicides totaux perpétrés en région rurale a atteint $54 \%{ }^{1}$.

Le recoupement entre la violence conjugale, la violence par arme à feu, et le fonctionnement du système de justice offre aux médecins des occasions d'infléchir le risque de féminicide au Canada par le biais d'interventions cliniques et de défense des intérêts. Même s'il faut s'intéresser autant aux meurtres commis à l'endroit des hommes aux plans des interventions et de la prévention, au Canada, ces homicides sont le plus souvent commis par des connaissances du milieu criminel ou autre, ou par de parfaits étrangers ${ }^{1,2,4}$; les occasions pour les médecins d'intervenir directement pour les prévenir sont moins évidentes. C'est en 2019 qu'on a commencé à noter l'identité de genre comme variable parmi les données sur les homicides au Canada. Puisqu'on ne s'entend pas, ni ici ni ailleurs dans le monde, sur la façon de rapporter le sexe et l'identité de genre en tant que variable, il est difficile de commenter les taux de féminicides et de violence conjugale chez les femmes trans au Canada actuellement.

\section{Points clés}

- Un féminicide se produit tous les 2,5 jours au Canada, souvent associé à la violence conjugale, et affecte de manière disproportionnée les femmes autochtones et les femmes vivant en zones non urbaines au Canada.

- Près de la moitié des femmes assassinées ont consulté un professionnel de la santé pour une blessure liée à la violence conjugale dans les 2 années précédant leur décès, ce qui indique que les médecins ont une occasion de reconnaître et de soutenir les personnes à risque.

- Il existe des ressources pour former les médecins à l'identification de la violence conjugale et les aider à s'informer sur la présence d'armes à feu à la maison, à gérer les questions de divulgation, à mettre au point des plans de sécurité et à contribuer à élaborer des programmes.

- Actuellement, la violence conjugale n'est pas spécifiquement reconnue comme infraction dans le Code criminel du Canada et les médecins ne peuvent pas se fier au système de justice pour assurer la sécurité de leurs patientes.

En 2018, l'Organisation des Nations Unies a révélé les conclusions d'une étude mondiale selon laquelle "le domicile est l'endroit le plus dangereux pour les femmes ${ }^{8}$. Les données canadiennes viennent étayer cette assertion. Entre 2016 et 2020, plus de $50 \%$ des victimes de féminicide au Canada ont été assassinées dans leur domicile et $83 \%-90 \%$ étaient mères ${ }^{1,3}$.

Fait à noter, $45 \%$ des femmes assassinées avaient consulté un professionnel de la santé pour une blessure liée à la violence conjugale au cours des 2 années précédant leur décès ${ }^{9}$. Les médecins ont donc des occasions de modifier la trajectoire de vie de ces femmes victimes de violence conjugale et qui sont exposées à un risque de féminicide ${ }^{7}$. Plusieurs ressources canadiennes, dont des plateformes éducatives et des outils validés, existent pour aider les médecins à reconnaître la violence conjugale, à gérer les enjeux de divulgation et à élaborer des programmes ${ }^{7}$. Le programme EDUCATE (www.ipveducate.com/the-educate-training -program) propose des ressources de formation multimodales à l'intention des chirurgiens et des cabinets médicaux. REAL Talk 
(Recognize, Empathize, Ask, Listen; https://realtalk.sagesse.org/) offre pour sa part des formations et des ateliers en ligne. Quant au programme Domestic Violence Education (http://dveducation.ca), on y trouve des modules d'apprentissage personnalisés que les médecins peuvent utiliser pour acquérir une compétence clinique. Un récent article rappelait les risques de violence conjugale à l'endroit des femmes durant le confinement imposé par la pandémie de COVID-19 et résumait quelques approches que les médecins pourraient mettre de l'avant pour s'attaquer au problème dans ce contexte ${ }^{7}$.

Au Canada, des rapports publiés montrent que 37\%-42\% des femmes et des filles assassinées l'ont été par arme à feu en 2019 et $2020^{1,5}$. On ne recueille pas et on n'a pas accès à des données sur les meurtres commis avec des armes à feu acquises légalement ou enregistrées ou avec des armes à feu ayant déjà fait l'objet de saisies. Il est donc impossible d'estimer l'effet des politiques d'enregistrement des armes à feu sur les féminicides. La présence d'une arme à feu dans un domicile multiplie par 5 le risque de létalité de la violence conjugale ${ }^{10}$. S'informer de la présence d'armes à feu à la maison peut aider les médecins canadiens à mettre sur pied un plan de sécurité dans les situations à risque. Les données d'autres territoires de compétence démontrent qu'il est acceptable, efficace et recommandé de s'informer auprès des patientes à risque de la présence d'armes à feu à la maison; un cadre proposé repose sur 5 éléments à retenir (l'arme qui se trouve au domicile est-elle chargée ou verrouillée, y a-t-il de jeunes enfants présents, l'utilisateur de l'arme à feu est-il déprimé et est-il conscientisé?) ${ }^{11}$. Il faut lancer et financer une recherche au Canada pour mesurer l'incidence de telles questions posées par les médecins au sujet des armes à feu.

Des données montrent toujours que des antécédents de violence masculine à l'endroit d'une partenaire sont le facteur de risque le plus important à l'égard du féminicide ${ }^{1,5,6,8,12}$. Une revue des homicides familiaux perpétrés en Ontario a révélé qu'entre 2003 et 2014, dans $72 \%$ des cas, l'agresseur avait déjà été violent à l'endroit de la victime ${ }^{12}$. Des études de cas au Canada montrent souvent que les agresseurs ont eu des démêlés avec la justice avant le meurtre ${ }^{5}$. Il est toutefois difficile de dégager des tendances, car le Code criminel du Canada ne prévoit pas d'infraction spécifiquement liée à la violence conjugale ${ }^{13}$. Les condamnations et les jugements pour violence conjugale ont de quoi inquiéter. En effet, selon une étude ontarienne sur les homicides, les hommes qui tuent leur conjointe ou des femmes de leur entourage reçoivent des sentences plus légères que les hommes qui commettent d'autres types de féminicide, ce qui semble témoigner d'une forme de "clémence liée à la proximité ${ }^{14}$. En outre, les comportements violents et agressifs à l'endroit des conjointes ne sont pas toujours suffisamment pris en compte pour modifier les décisions du Tribunal de la famille au Canada, par exemple, dans les cas de garde d'enfants ${ }^{1}$.

Le Projet de loi C-3, qui vise à amender la Loi sur les juges et le Code criminel, a reçu la sanction royale et est entré en vigueur en 2021. Elle oblige les juges de la Cour supérieure à suivre une formation sur les agressions sexuelles et inclut des amendements qui spécifient que le contexte social inclut le racisme systémique et la discrimination. Or, on n'a pas jugé bon d'inclure les amendements suggérés par l'ombudsman fédéral des victimes d'actes criminels relativement au phénomène d'intersectionnalité dans la violence domestique. De plus, cette loi ne s'applique pas aux juges des cours provinciales qui entendent la majorité des causes de violence sexuelle et en droit de la famille (qui concernent la protection de la jeunesse, la garde des enfants et les droits d'accès) au Canada; la formation devrait être obligatoire pour tous les juges appelés à se prononcer sur les cas de violence conjugale.

Les médecins devraient s'allier aux responsables des orientations politiques pour que le Code criminel canadien soit plus transparent au chapitre de la violence conjugale, que la collecte de données soit plus efficace et qu'on améliore la qualité des rapports sur les crimes par arme à feu afin que l'on sache quel est le type d'arme, l'endroit où elle se trouve, qui en est propriétaire et si elle est enregistrée. Il faut également mettre des programmes sur pied et les offrir à tous les niveaux de la formation médicale pour enseigner aux professionnels de la santé comment reconnaître et soutenir les femmes exposées à un risque de violence conjugale.

Le domicile ne devrait pas continuer d'être l'endroit le plus dangereux pour les femmes ${ }^{8}$ et les médecins canadiens ne peuvent pas simplement se fier au système de justice pour veiller à la sécurité de leurs patientes et prévenir des décès dus à la violence conjugale perpétrée par des conjoints ou des exconjoints. Comme médecins et défenseurs des intérêts des femmes, nous avons du travail à faire pour promouvoir les changements qui feront du Canada un endroit plus sécuritaire pour les femmes et les filles.

\section{Références}

1. \#CallItFemicide: understanding sex/gender related killings of women and girls in Canada, 2020. CFOJA reports. Guelph (ON): Canadian Femicide Observatory for Justice and Accountability (CFOJA); 2020. Accessible ici : https:// femicideincanada.ca/callitfemicide2020.pdf (consulté le 25 juin 2021).

2. Table 35-10-0156-01: Number, percentage and rate of homicide victims, by gender and Indigenous identity. Ottawa: Statistics Canada. Accessible ici : https://www150. statcan.gc.ca/t1/tbl1/en/tv.action?pid=3510015601 (consulté le 15 juin 2021).

3. \#CallItFemicide: 2021 mid-year report. Guelph (ON): Canadian Femicide Observatory for Justice and Accountability (CFOJA); 2021.

4. Moreau G, Jaffray B, Armstrong A; Canadian Centre for Justice and Community Safety Statistics. Police-reported crime statistics in Canada, 2019. Ottawa: Statistics Canada; modified 2020 Oct. 29. Accessible ici : https://www150.statcan. gc.ca/n1/pub/85-002-x/2020001/article/00010-eng.htm (consulté le 2 juin 2021).

5. \#CallltFemicide: understanding gender related killings of women and girls in Canada 2019. Guelph (ON): Canadian Femicide Observatory for Justice and Accountability (CFOJA); 2019. Accessible ici : https://www.femicideincanada. ca/callitfemicide2019.pdf (consulté le 24 juin 2021).

6. Eighteen months after leaving domestic violence is still the most dangerous time. Vancouver: Battered Women's Support Services; 2020. Accessible ici : https://www.bwss.org/eighteen-months-after-leaving-domestic-violence-is-still -the-most-dangerous-time/ (consulté le 3 juin 2021).

7. Bradley NL, DiPasquale AM, Dillabough K, et al. Health care practitioners' responsibility to address intimate partner violence related to the COVID-19 pandemic. CMAJ 2020;192:E609-10

8. Home, the most dangerous place for women, with majority of female homicide victim murdered by partners of family UNODC study says [communiqué de presse]. Vienna [Austria]: United Nations Office on Drugs and Crime; 2018 Nov. 25. Accessible ici : https://www.unodc.org/unodc/en/press/releases/2018/ November/home--the-most-dangerous-place-for-women--with-majority-of -female-homicide-victims-worldwide-killed-by-partners-or-family--unodc-study -says.html (consulté le 14 juin 2021). 
9. Bhandari M, Dosanjh S, Tornetta P III, et al.; Violence Against Women Health Research Collaborative. Musculoskeletal manifestations of physical abuse after intimate partner violence. J Trauma 2006;61:1473-9.

10. Campbell JC, Webster D, Koziol-LcLain SJ, et al. Risk factors for femicide in abusive relationships: results from a multisite case control study. Am J Public Health 2003;93:1089-97.

11. Pallin R, Spitzer SA, Ranney ML, et al. Preventing firearm-related death and injury. Ann Intern Med 2019;170:ITC81-96.

12. Domestic Violence Death Review Committee: 2013-14 annual report. Toronto: Office of the Chief Coroner for Ontario; 2015. Accessible ici : https://www.mcscs. jus.gov.on.ca/sites/default/files/content/mcscs/docs/ec165340.pdf (consulté le 6 juin 2021).

13. Cullen P, Dawson M, Price J, et al. Intersectionality and invisible victims: reflections on data challenges and vicarious trauma in femicide, family and intimate partner homicide research. J Fam Violence 2021;36:619-28.

14. Dawson M. Punishing femicide: criminal justice responses to the killing of women over four decades. Curr Sociol 2016;64:996-1016.

Intérêts concurrents : Najma Ahmed déclare être membre du Conseil d'administration de Médecins canadiens pour un meilleur contrôle des armes à feu. La $D^{\text {re }}$ Ahmed est aussi membre de l'Association canadienne de traumatologie et de l'American College of Surgeons, organismes qui militent pour la prévention de la violence par arme à feu. Aucun autre intérêt concurrent n'a été déclaré.

Cet article a été soumis à l'examen des pairs.

Affiliations : Département de chirurgie (Bradley, Gawad), Université de l'Alberta, Edmonton, Alb.; Département de médecine de soins intensifs (Bradley), Université de la Colombie-Britannique, Vancouver, C.-B.; Olthuis Kleer Townshend LLP (McNamara); Département de chirurgie (Ahmed), Université de Toronto, Toronto, Ont.

Collaborateurs : Tous les auteurs ont contribué à l'élaboration et à la conception des travaux ainsi qu'à l'acquisition, à l'analyse et à l'interprétation des données. Tous les auteurs ont participé à la rédaction du manuscrit, en ont révisé de façon critique le contenu intellectuel important, ont donné leur approbation finale pour la version destinée à être publiée, et assument l'entière responsabilité de tous les aspects du travail.

Propriété intellectuelle du contenu : Il s'agit d'un article en libre accès distribué conformément aux modalités de la licence Creative Commons Attribution (CC BY-NC-ND 4.0), qui permet l'utilisation, la diffusion et la reproduction dans tout médium à la condition que la publication originale soit adéquatement citée, que l'utilisation se fasse à des fins non commerciales (c.-à-d., recherche ou éducation) et qu'aucune modification ni adaptation n'y soit apportée. Pour plus de renseignements, consulter le https://creativecommons.org/ licenses/by-nc-nd/4.0/deed.fr.

Correspondance : Nori Bradley, nlbradle@ualberta.ca 